\title{
田HAD
}

DOI: http://doi.org/10.22585/hospdomic.v1i1.2

\section{Tratamiento domiciliario de las metástasis cutáneas de carcinoma mamario por parte del equipo de cuidados paliativos}

\section{Home treatment of skin metastases of breast carcinoma by the palliative care team}

Andrea Illán', Teresa Galea², Victoria Romero², Rocío Valentín², Patricia Hernández ${ }^{2}$, Fabiola Saiz², Ángel María Duran², Ángel Domínguez ${ }^{3}$

1. Servicio de Oncología Médica del Hospital San Pedro de Alcántara (Cáceres), España.

2. Unidad de Cuidados Paliativos del Hospital San Pedro de Alcántara (Cáceres), España.

3. Servicio de Medicina Interna del Hospital San Pedro de Alcántara (Cáceres), España.

Correspondencia/Correspondence

andrea88med@yahoo.es

RECIBIDO/RECEIVED

28.09.2016

ACEPTADO/ACCEPTED

27.11 .2016
CONFLCTO DE INTERESES/COMPETING INTEREST Los autores de este trabajo declaran la inexistencia de cualquier tipo de conflicto de interés.

FINANCIACIÓN/FUNDINGS

No declara ninguna fuente específica de financiación ni ayuda económica recibida. 


\section{RESUMEN}

Presentamos el caso clínico de una paciente de 70 años diagnosticada de cáncer de mama metastásico, que presentó ulceración de probables metástasis cutáneas de novo. El reconocimiento y abordaje terapéutico adecuado de las complicaciones de las metástasis cutáneas es indispensable en el cuidado y control de síntomas del paciente incluido en programa de cuidados paliativos o unidad de hospitalización a domicilio.

Palabras clave: metástasis cutáneas; úlceras tumorales; metástasis cáncer mama.

\section{ABSTRACT}

We report the case of a female patient aged 70 , with the diagnosis of metastatic breast cancer, who presented ulceration of possible skin metastases de novo. The recognition and appropriate therapeutic approach of complications of skin metastases is essential in the care and control of patient's symptoms included in palliative care program or home hospitalization unit.

Palabras clave: Breast Neoplasms; Neoplasm Metastasis; Ulcer; Palliative Care; Home Care Services, Hospital-Based; Aged. 


\section{INTRODUCCIÓN}

Las metástasis cutáneas suponen el 2\% de los tumores cutáneos. La sobreinfección, hemorragia y la ulceración son las complicaciones más frecuentes. Resulta fundamental conocer el manejo para un abordaje óptimo e integral del paciente paliativo.

\section{DESARROLLO DE LA EXPERIENCIA CLÍNICA}

Presentamos el caso clínico de una mujer de 70 años con carcinoma ductal infiltrante de mama con metastatización pulmonar y hepática múltiple, en tratamiento con hormonoterapia paliativa por rechazar tratamiento citostático. Tras un intervalo libre de progreso superior a 36 meses, la paciente acudió acompañada de su cuidadora principal a urgencias de nuestro hospital por bultomas cutáneos y síndrome constitucional. La exploración física reveló caquexia y la presencia pericicatriz de mastectomía derecha de una masa nodular ulcerada no sangrante de $5 \times 5 \mathrm{~cm}$ adherida a piel, y otras dos masas no ulceradas y de las mismas características en FID y tercio superointerno de muslo izquierdo. Existía anemia microcítica e hipocroma, leve leucocitosis con desviación izquierda, con exámenes de coagulación y bioquímica dentro de la normalidad. La radiografía de tórax mostró imagen en suelta de globos compatible con metastatización pulmonar múltiple. El Equipo de Cuidados paliativos fue avisado por el servicio de urgencias y programó visita domiciliaria. Se reexploró a la paciente, encontrándose como hallazgos principales lesiones nodulares sobre elevadas, ulceradas y dolorosas a la palpación (Figuras 1 y 2), con claros signos físicos de infección local. Enfermería aplicó fórmula magistral con metronidazol y lidocaína, con mejoría clínica de las lesiones.

Dados los hallazgos exploratorios y en el contexto clínico del paciente, la hipótesis diagnóstica giró en torno a metástasis cutáneas múltiples de carcinoma mamario. La paciente rechazó realización de biopsia cutánea confirmatoria del diagnóstico.

Hemos realizado una revisión de la literatura científica acerca del manejo de las úlceras de origen tumoral a propósito del caso clínico descrito.

La metástasis cutáneas suponen el 2\% de los tumores cutáneos, representando entre el 0,6 y al 10,4\% de todas las metástasis. En mujeres el tumor que de forma más frecuente las produce es el cáncer de mama (1-3) seguido del cáncer de ovario o intestino delgado y del melanoma, según diferentes autores. En hombres, encabeza la etiología el cáncer de pulmón, seguido de intestino delgado y de melanoma (éste último debido a su epidermotropismo).

1. Hallazgos exploratorios a la visita domiciliaria

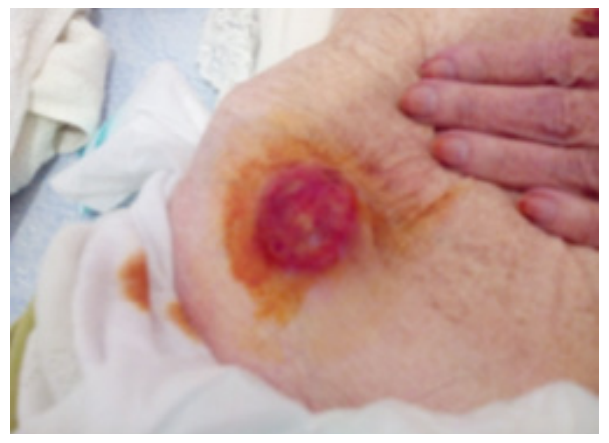

2. Hallazgos exploratorios a la visita domiciliaria

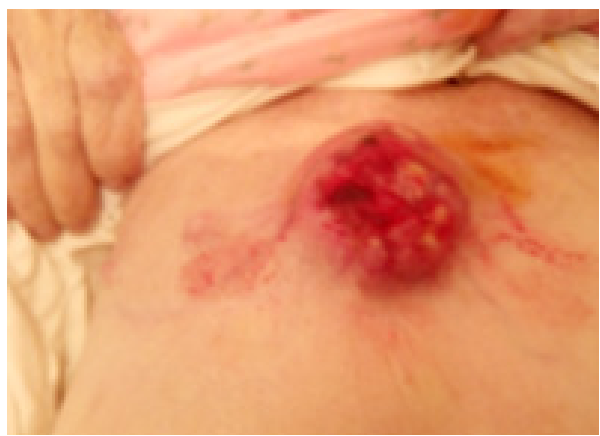


Las metástasis de cáncer de mama pueden presentarse clínicamente según Schwartch de siete formas diferentes: forma nodular (la más frecuente), forma, inflamatoria o erisipeloide, telangiectásica, en coraza o esclerodermiforme, del pliegue inframamario o alopecia neoplásica. Para algunos autores, el tronco es la localización más frecuente para las metástasis cutáneas de neoplasias de mama.

El diagnóstico diferencial de las lesiones cutáneas es variado. En las pacientes con cáncer de mama, se deben de considerar entre otras entidades: los cambios cutáneos postratamiento (p.ej, rádicos) así como procesos infecciosos como erisipela, celulitis o candidiasis. Es necesaria la realización de biopsia cutánea para su diagnóstico definitivo. Al indicar la aparición de metástasis cutáneas una enfermedad avanzada, los potenciales pilares de tratamiento se basan en radioterapia, quimioterapia sistémica o tópica (parecen resultar útiles cremas de miltefosina 6\%, imiquimod 5\%) y hormonoterapia paliativas.

En cuanto a la limpieza de la herida, se recomienda emplear suero salino fisiológico, especialmente si hay afectación de tejidos y estructuras profundas. En curas frecuentes se deben evitar cintas adhesivas o vendajes, siendo preferible utilizar mallas de sujeción tubulares. El desbridamiento estará indicado en caso de tejido necrótico. Hay que preparar cuidadosamente la zona perilesional, para asegurar un buen sellado, protegerla con cremas barrera o películas protectoras para evitar la maceración.

Las complicaciones más frecuentes de las metástasis cutáneas son la sobreinfección, hemorragia y la ulceración. Ante la sospecha de infección, debe pautarse antibióticos vía tópica u oral. Para evitar sangrado, se pueden emplear apósitos antiadherentes y realizar una limpieza suave de la úlcera por irrigación y si no es suficiente, valorar la administración de agentes hemostáticos tópicos.

Se pueden utilizar agentes antihistamínicos para prurito.

Ante el dolor, se deben seguir las pautas analgésicas según la OMS (escalera o ascensor analgésico), pudiendo ser necesario en algunos casos analgesia pautada 20 - 30 minutos antes de realizar la cura, incluso pudiéndose combinar con sedación.

\section{CONCLUSIONES}

El reconocimiento y abordaje terapéutico adecuado de las complicaciones de las metástasis cutáneas, es indispensable en el cuidado y control de síntomas del paciente oncológico con enfermedad avanzada. El equipo de Cuidados Paliativos o Unidad de Hospitalización a domicilio deben estar formados en dicha identificación y manejo sintomático de las metástasis cutáneas.

\section{BIBLIOGRAFÍA}

1. Gómez Sánchez ME, Martínez Martínez ML, Martín De Hijas MC, López Villaescusa MT, Faura Berruga MC, Rodríguez Vázquez M, et al. Metástasis cutáneas de tumores sólidos: Estudio descriptivo retrospectivo. Piel (Barc). 2014;29(4):207-12. DOI: 10.1016/j.piel.2013.11.007

2. Torres-Aja L. Metástasis cutánea de carcinoma mamario: cáncer de mama en coraza; revisión de la literatura y presentación de un caso. Rev Finlay [revista en Internet]. 2012 [citado 1 septiembre 2016]; 2(3):[aprox 5 pantallas]. Disponible en: http://www.revfinlay.sld.cu/index.php/ finlay/article/view/121

3. Nava G, Greer K, Patterson J, Lin KY. Metastatic cutaneous breast carcinoma: a case report and review of the literature. Can J Plast Surg. 2009;17(1):25-7. PMID: 20190910 